\title{
Cost-Effectiveness Analysis of Breast Cancer Screening in Rural Iran
}

\author{
Nooshin Zehtab ${ }^{1}$, Mohammad Jafari ${ }^{2}$, Mohsen Barooni ${ }^{3}$, Nouzar Nakhaee ${ }^{4}$, Reza \\ Goudarzi $^{5 *}$, Mohammad Hassan Larry Zadeh ${ }^{6}$
}

\begin{abstract}
Background: Although breast cancer is the most common cancer in women, economic evaluation of breast cancer screening is not fully addressed in developing countries. The main objective of the present study was to analyze the cost-effectiveness of breast cancer screening using mammography in 35-69 year old women in an Iranian setting. Materials and Methods: This was an economic evaluation study assessing the cost-effectiveness of a population-based screening program in 35-69 year old women residing in rural areas of South east Iran. The study was conducted from the perspective of policy-makers of insurance. The study population consisted of 35- to 69-year old women in rural areas of Kerman with a population of about 19,651 in 2013. The decision tree modeling and economic evaluation software were used for cost-effectiveness and sensitivity analyses of the interventions. Results: The total cost of the screening program was 7,067.69 US\$ and the total effectiveness for screening and no-screening interventions was 0.06171 and 0.00864 disability adjusted life years averted, respectively. The average cost-effectiveness ratio DALY averted US\$ for screening intervention was 7,7082.5 US\$ per DALY averted and 589,027 US \$ for no-screening intervention. The incremental cost-effectiveness ratio DALY averted was 6,264 US\$ per DALY averted for screening intervention compared with no-screening intervention. Conclusions: Although the screening intervention is more cost-effective than the alternative (noscreening) strategy, it seems that including breast cancer screening program in health insurance package may not be recommended as long as the target group has a low participation rate.
\end{abstract}

Keywords: Cost-effectiveness - screening - breast cancer - DALY averted

Asian Pac J Cancer Prev, 17 (2), 609-614

\section{Introduction}

Breast cancer is the most common cancer among women worldwide (Alsanabani et al., 2015). According to GLOBOCAN report in 2012 , breast cancer was the most common cancer in both developed and developing countries in 2012. According to this report, the incidence of breast cancer is 27 in hundred thousands of women in Africa, Middle East, and East Asia to 96 per hundred thousands of women in Western Europe (GLOBOCAN, 2012).

Middle and low income countries will bear the highest damage from non-communicable diseases and poor people are more vulnerable in this regard. Inadequate and limited access to health care is a major cause of this vulnerability (Haghighat, 2013). Since the average age is lower in developing countries, such as Iran, the costs of lost production due to premature death increases; therefore interventions should be adopted to more effectively treat the patients, improve their survival, and increase the effectiveness (Davari et al., 2013). According to the World Health Organization (WHO), early detection of breast cancer is an effective solution to improve health outcomes, because early detection of early-stage breast cancer reduces mortality and costs (Anderson et al., 2008; Nguyen et al., 2013).

Mammography screening is a way to reduce mortality from breast cancer in women aged 40 and higher (Greif, 2013). Screening programs aim at detecting the disease after initiation and before leading to clinical symptoms (Khalili et al., 1999).

In the current situation of the community, lower-cost strategies with maximum effectiveness are certainly very important for policymakers of the health sector (Nahvijou et al., 2014). Based on cost-effectiveness, due to limited resources as well as concerns associated with justice for health system, the importance of decisions about interventions of health and treatment is increasing every day.

Several studies have been done in this field, including

${ }^{1}$ Health Economics, Social Determinants of Health Research Center, ${ }^{2}$ Kerman province health insurance, ${ }^{3}$ Health Economics, Research center for modeling in health, ${ }^{5}$ Health Economics, Health Services Management Research Center, ${ }^{4}$ Community Medicine, Neuroscience Research Center, Institute of Neuropharmacology, ${ }^{6}$ Department of Radiotherapy and Oncology, Kerman University of Medical Sciences, Kerman, Iran*For correspondence: rgoudarzi@yahoo.com 
the study of Reza et al. (2014) systematic review study (Reza et al., 2014), the study by Laurens et al. (2014) in Central America: The cases of Costa Rica and Mexico (Laurens et al., 2014) and the study of Ki-Bong et al. (2013) entitled "Is mammography for breast cancer screening cost effective in both Western and Asian countries?: Results of a systematic review" (KI-BONG et al., 2013).

In the present study, breast cancer screening program using mammography was compared with no-screening in terms of cost-effectiveness.

\section{Materials and Methods}

This is an economic evaluation. The perspective of the study was to consider the costs and outcomes of policymakers of the insurance organization. The study population consisted of 35 to 69 years old woman in rural areas of Kerman with a population of about 19,651 people in 2013.

In this study, all people were screened through public screening using a census method and the data were collected with the risk assessment questionnaire. Screening was conducted in three phases, the first phase: risk assessment with the questionnaire, the second phase: identification of the persons eligible for mammography, and the third phase: selective screening with mammography which details and mode of screening was mentioned by Jafari et al. (Jafari et al., 2015). Costeffectiveness analysis and sensitivity analysis of both interventions was performed using the decision tree model and TreeAge 2011. The study has been approved by the University's ethics committee.

\section{Decision analysis model}

After screening, the decision tree model was designed for cost-effectiveness analysis in which two options of screening and no-screening were compared. In this model, 3000 people were included in the screening intervention and 12,794 people were enrolled in the no-screening intervention. (Figure 1) shows the modeling used in this study.

\section{Model inputs}

Epidemiological data and the possibilities were obtained from the reports of WHO, scientific literature, and valid domestic and foreign resources. This included the prevalence of breast cancer in Iran, the sensitivity and specificity of mammography and ultrasound, DALY, and the possibility of diagnosis stage of breast cancer in the absence of screening.

(Table 1) shows the base cases used in the model and the range of each possibility, and the scope of each possibility was used for sensitivity analysis.

\section{Effectiveness}

In this model, the DALY averted was considered as the outcome and effectiveness of intervention.

$$
\mathrm{DALY}=\mathrm{YLL}+\mathrm{YLD}
$$

\section{YLL=}

$$
\frac{\mathrm{KCe}}{(\mathrm{r}+\beta)^{2}}\left[\mathrm{e}^{-(\mathrm{r}+\beta)(\mathrm{L}+\mathrm{a})}\{-(\mathrm{r}+\beta)(\mathrm{L}+\mathrm{a})-1\}-\mathrm{e}^{-(\mathrm{r}+\beta)}\{-(\mathrm{r}+\beta) \alpha-1\}\right]+\frac{1-\mathrm{K}}{\mathrm{r}}\left(1-\mathrm{e}^{-\mathrm{rL}}\right)
$$

\section{YLL=}

$\mathrm{D}\left[\frac{\mathrm{KCe}}{(\mathrm{r}+\beta)^{2}}\left[\mathrm{e}^{-(\mathrm{r}+\beta)(\mathrm{L}+\mathrm{a})}\{-(\mathrm{r}+\beta)(\mathrm{L}+\mathrm{a})-1\}-\mathrm{e}^{-(\mathrm{r}+\beta) a}\{-(\mathrm{r}+\beta) \alpha-1\}\right]+\frac{1-\mathrm{K}\left(1-\mathrm{e}^{-\mathrm{rL}}\right)}{\mathrm{r}}\right]$

Where $\mathrm{K}$ is the constant combined age-weighting (1), $\beta$ the constant age-weighting (0.04), $\mathrm{C}$ the adjusted ageweighting (0.1658), e the base of natural logarithm, D the disability weight, $r$ the discount rate $(0.03)$, $L$ the average length of treatment (in years) in YLD and the crude lost years of YLL, and a is the disease onset in age group in YLD and age at death in YLL (WHO, 2003).

\section{Costs}

In this model the direct costs of breast cancer were evaluated for the screening and the no-screening intervention. Direct costs of screening included the costs of mammography, interpretation of the results, ultrasound, biopsy, and consumables, treatment, labor, advertising, support, transportation, printing and reproduction of questionnaires, and data entry. The costs of screening intervention are shown separately in (Table 2). The costs of direct treatment of cancer in the no-screening intervention were separately calculated based on a discount rate of $3 \%$, $5 \%$, and $7 \%$ for the 4 stages of cancer diagnosis from the average costs of private and public sectors (Tables 3 ). Due to the lack of sufficient data, analysis of indirect costs (including travel, disability, low productivity, pain-related psychological costs, reduced efficiency, and premature

Table 1. The Basic Cases used in the Cost-effectiveness Analysis Model of Screening and No-screening Interventions

\begin{tabular}{lrrl}
\hline Variable & Value & Range & References \\
\hline Mammography sensitivity & 0.73 & $0.55-0.9$ & (Mousavi et al., 2009; Akbari et al., 2012) \\
Mammography specificity & 0.45 & $55.3-0.97$ & (Mousavi et al., 2009; Akbari et al., 2012) \\
Ultrasound sensitivity & 0.69 & $0.63-0.9$ & (Mousavi et al., 2009; Akbari et al., 2012) \\
Ultrasound specificity & 0.49 & $0.49-50.7$ & (Mousavi et al., 2009; Akbari et al., 2012) \\
Breast cancer prevalence (per 100,000 people) & 120 & $86-129$ & (Akbari et al., 2012; WHO, 2008) \\
Possibility of detection at stage I & 0.041 & $0.0369-0.15$ & (Davari et al., 2013, Groot et al., 2006) \\
Possibility of detection at stage II & 0.282 & $0.141-0.52$ & (Davari et al., 2013, Groot et al., 2006) \\
Possibility of detection at stage III & 0.266 & $0.1-0.58$ & (Davari et al., 2013, Groot et al., 2006) \\
Possibility of detection at stage IV & 0.411 & $0.184-0.4521$ & (Davari et al., 2013, Groot et al., 2006) \\
DALY averted in the first stage of treatment & 19.25 & $12.25-23.41$ & (Groot et al., 2006) \\
DALY averted in the second stage of treatment & 3.66 & $2.24-4.13$ & (Groot et al., 2006) \\
DALY averted in the third stage of treatment & 1.63 & $1.60-1.74$ & (Groot et al., 2006) \\
DALY averted in the fourth stage of treatment & 0.18 & $0.16-0.19$ & (Groot et al., 2006) \\
\hline
\end{tabular}


death) was not performed. Cost is calculated on the basis of US dollars in 2013.

By comparing the relative costs and outcomes of two interventions, the incremental cost-effectiveness ratio (ICER) was calculated. ICER has been defined as the ratio of change in costs to change in effects of a particular intervention compared with an alternative intervention (Carles et al., 2011), and the average cost-effectiveness (ACER) of the cost to effectiveness ratio of an intervention was calculated without a reference for comparison (Bang \& Zhao, 2012). The cost and effectiveness was entered in the model considering the discount rate of 0.05 (MahboubAhari et al., 2014).

ICER $=[($ Cost Screening - Cost No Screening $) /($ DALY averted Screening - DALY averted No Screening)]

The cost-effectiveness of screening was then evaluated with regard to ICER and ACER obtained in the mentioned program and comparing them with the threshold set by WHO for developing countries (3 times of per capita GDP) (Eichler et al., 2004).

\section{Sensitivity analysis}

For sensitivity analysis, the tornado diagram was drawn according to which sensitivity analysis was performed for the parameters with the greatest impact on the cost-effectiveness analysis. The sensitivity analysis in this study was performed for parameters such as sensitivity and specificity of mammography and ultrasound diagnostic methods, DALY, prevalence of breast cancer in Iran, possibility of eligibility, and possibility of further follow-up with mammography and ultrasound and costs through one-way and two-way methods. The range was obtained either from other studies for some parameters or through $10 \%$ changes in parameters (Sari et al., 2013).

\section{Results}

Among the 19,651 participants, 15,794 questionnaires were completed by women, of whom 3,000 were included in the screening intervention and 12,794 people in the no-screening intervention.

(Table 2) shows the costs of screening program in separate. To calculate the cost of each branch of the decision tree model, the cost per person was obtained. The highest and lowest costs were related to mammography and duplication of questionnaires.

(Table 3) depicts the costs of cancer treatment in the no-screening intervention. These costs are different for each stage of the cancer.

The results in Table 4 indicate that the total effectiveness of the screening and no-screening interventions was 0.06171 and 0.00864 DALY averted, respectively. The average cost-effectiveness (C/E) ratio was 77082.5 dollars per DALY averted for the screening intervention and 589,027 dollars per DALY averted for the no-screening intervention. Given that the smaller the $\mathrm{C} / \mathrm{E}$ ratio, the greater would be the cost-effectiveness, the screening intervention is more cost-effective in this perspective. The incremental cost-effectiveness ratio was 6264 dollars per
DALY averted for the screening intervention compared



Figure 1. Decision Tree Modeling for the CostEffectiveness of Breast Cancer Screening

Table 2. Costs of the Screening Program (US \$)

\begin{tabular}{lrc}
\hline Cost title & Total cost & Per capita cost \\
\hline Mammography & 13.55 & 13.55 \\
Ultrasound & 3.69 & 3.69 \\
Biopsy & 9.01 & 1.12 \\
Biopsy consumables & 90.4 & 11.3 \\
Questionnaire completion & 5353.79 & 0.33 \\
Questionnaire duplication & 133.84 & 0.008 \\
Advertizing and support & 338.97 & 0.021 \\
Transportation & 338.97 & 0.11 \\
Data entry & 446.5 & 0.028 \\
Unexpected & 338.97 & 0.11 \\
\hline
\end{tabular}

Table 3. Costs of the No-screening Intervantion (US \$)

\begin{tabular}{lrc}
\hline Stage & Per capita cost & References \\
\hline Treatment of stage I & 1669.59 & (Davari et al., 2013) \\
Treatment of stage II & 11957.34 & (Davari et al., 2013) \\
Treatment of stage III & 14929.67 & (Davari et al., 2013) \\
Treatment of stage IV & 22132.76 & (Davari et al., 2013) \\
\hline
\end{tabular}

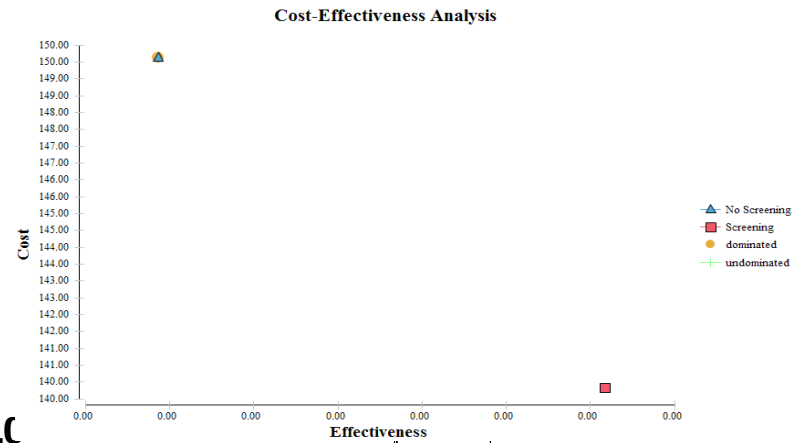

Figure 2. Cost-effectiveness Ratios on the CostEffectiveness Plane



Figure 3. Tornado Diagram for Sensitivity Analysis 
Table 4. The Incremental Cost-effectiveness Ratio of Both Interventions in (costs in dollars)

\begin{tabular}{lcccccc}
\hline Intervention & Effectiveness & Incremental effectiveness & Cost & Incremental Cost & ACER & ICER \\
\hline Screening & 0.00864 & --- & 5089.1 & -- & 589027 & -- \\
No-screening & 0.06171 & 0.05307 & 4756.76 & -332.34 & 77082.5 & -6264 \\
\hline
\end{tabular}

Table 5. Comparison of Cost-effectiveness with Per Capita GDP

\begin{tabular}{lcccc}
\hline & $\begin{array}{c}\text { ICER /per } \\
\text { capita GDP }\end{array}$ & $\begin{array}{c}\text { ICER /3 times } \\
\text { per capita GDP }\end{array}$ & $\begin{array}{c}\text { ACER/per } \\
\text { capita GDP* }\end{array}$ & $\begin{array}{c}\text { ACER/3 times per } \\
\text { capita GDP** }\end{array}$ \\
\hline $\begin{array}{l}\text { Screening program } \\
\text { No-screening program }\end{array}$ & 1.32 & 0.43 & 16.18 & 5.39 \\
\hline
\end{tabular}

*per capita GDP : 4763.3 US \$; ** 3 times per capita GDP: 14289.9 US \$

with the no-screening intervention.

(Figure 2) shows the cost-effectiveness analysis in which the no-screening intervention is located at the undominated region and costs are higher than threshold of 3 times of per capita GDP and are not cost-effective.

(Table 5) shows the ratio of ACER and ICER to per capita GDP and 3 times per capita GDP. The ratio of ACER to per capita GDP and 3 times per capita GDP is higher than one. The ratio of ICER to 3 times per capita GDP is less than one.

The cost of breast cancer treatment at any stage of diagnosis obtained from the average costs of private and public sectors. The discount rates $3 \%$ and $7 \%$ were used (Mahboub-Ahari et al., 2014) in the sensitivity analysis, that did not affect the results.

\section{Discussion}

Cost-effectiveness analysis can provide useful information for planning and development of a breast cancer control policy. It can also be used to allocate scarce resources to national programs of breast cancer control and to identify the most efficient way to provide diagnostic and treatment services. Almost all studies on the costs and health effects of breast cancer control interventions have been conducted in developed countries, but information for decision-making regarding resource allocation in developing countries are scarce. According to the results in Table 5, screening intervention is more cost-effective in terms of $\mathrm{C} / \mathrm{E}$ perspective. ICER of breast cancer screening with mammography was 6264 dollars per DALY averted in comparison with the no-screening intervention. In general, the no-screening intervention per DALY averted was 333 dollars more expensive than the screening intervention, i.e. if nobody is screened, an amount of 333 dollars will be imposed on the insurance company in the future per person. According to the study of Mandelblatt et al., of the 116 women screened for breast cancer, the average cost of the program was high: about 1,829 dollars per patient which is expensive given the low number of women screened for breast cancer (Mandelblatt et al., 1997). This study had a high cost similar to our study.

In California, the cost-effective strategy to begin screening at age 40 was not effective but biennial screening with mammography from age 50 was costeffective. This policy maximizes the cost-effectiveness of mammography screening program with limited budget.
This finding is not surprising because the prevalence of breast cancer in women aged 50 and older is high (Melnikow et al., 2013). In this study, screening was started from the age 35 but given the low participation of women in the screening program, one could not accurately determine whether selecting a lower age for screening programs is cost effective?

The effectiveness of this study was low, a reason of which may be the low effectiveness of short interval of screening (1 year). In the study by Rojnik et al., screening interval of 1 year in breast cancer screening had fewer benefits and higher costs compared with screening interval of 2 years. Therefore, the optimal screening policy should be adopted from the policies with 3 years interval (Rojnik et al., 2008). This study confirms our results.

One of the results of screening programs is decreased mortality which was not investigated in this study because of the short interval of the screening program; however, in a study in India, Biennial screening with mammography in simulation results in a greater reduction in mortality and increases the number of life-years saved, but the costs have increased a lot (Lamberts et al., 2008).

According to Table 5, the ratio of ACER to per capita GDP is more than one; i.e. the cost per DALY averted is higher than per capita GDP. If CE/per capita GDP is higher than 1 , screening with mammography can be considered ineffective because the cost per LYS (life-years saved), QALY or DALY is higher than per capita GDP (KI-BONG et al., 2013). The ratio was 16.18 for screening program and 123.65 for no-screening intervention. Although the ratio of both interventions was more than 1 in Iran, suggesting the ineffectiveness of mammography screening in the country, the difference between the two ratios shows that screening in Iran is better than no-screening, and not screening will face the country with additional costs. Our results were similar to many Asian countries such as, where CE/per capita GDP is more than 1 and not cost-effectiveness. In contrast, it is cost-effective in the West; the ratio of CE/per capita GDP is less than 1. As an exception, the CE/per capita GDP ratio in Japan was about 0.85 in comparison with other Asian countries. The incidence of breast cancer in Japan is rapidly increasing, and it has currently the highest rate among all women's cancers (Yip et al., 2008). One possible reason for the difference in the cost-effectiveness of mammography screening for breast cancer between Asian countries and western countries is different incidence, and this difference 
underscore the need to consider the incidence when using mammography screening. When the incidence of breast cancer is high, breast cancer is more likely to be diagnosed by mammography; thus, in countries where the incidence is high, more life-years are saved in comparison with countries with lower incidence (KI-BONG et al., 2013).

In Iran, ICER of 3 times per capita GDP and the threshold considered for low-income countries by WHO (Mahboub-Ahari et al., 2014) is low; and taking this perspective, one can say that breast cancer screening is cost-effective. In South Korea, ICER was slightly higher than per capita GDP; about 27,168 US \$ in 2009. In the United States, additional costs for screening of 50-69 years women was 37,000 dollars per LYS, indicating about $87 \%$ of per capita GDP (Hae et al., 2013). In most developed countries with breast cancer screening programs, the estimated ICER is less than per capita GDP. For example, the costs of treatment in the organized breast cancer screening with mammography in Switzerland were 14,452 per LYS dollars which was $26 \%$ of per capita GDP of Switzerland (De Gelder et al., 2009).

Sensitivity analysis showed that the costs and changes in possibilities had no impact on the result achieved. Sensitivity analysis in other studies showed that small and medium changes in model inputs, did not affect the results (Carles et al., 2011). Also different model assumptions affected cost effectiveness but not affected the main results of the study (Laurens et al., 2014) which confirm the results of our study.

This study had some limitations, for example the problem of arrangement between the administrative units, lack of appropriate models for breast cancer screening in Iran, requirement of skill, expertise, and experience of radiologist in the diagnosis of disease, and lack of qualified doctors in the plan, impossibility of using the media to raise awareness, difficulty of monitoring the implementation of the screening program because of scattered villages and cities under 20 thousand people, low participation of qualified individuals in screening programs (34\%), not easy to predict the outcome of the screening program due to the first screening experience in the country, the small size of the sample for screening, and short-term of follow-up of eligible participants in the program (one year).

In conclusion, Since the participation of target groups is one of the prerequisites of success of screening programs and low participation level for a screening program is undesirable and leads to reduced efficacy, media group, operators of public education, especially health organizations, insurance agencies have an important responsibility in this regard. They should improve participation in screening programs to identify cancer at early stages. Although the screening intervention is more cost-effective than no-screening, it seems that including breast cancer screening program in the health insurance package is not a good idea as long as the target group has a low possibility of participation, because the costeffectiveness of the program would be too low.
The authors would like to give their gratitude to the Iranian Health Insurance Organization, which approved this research and assumed the implementation costs aiming at improving women's health in Kerman Province, and to the Health Deputy of Medical Sciences University which tried honestly for implementation of the study with all facilities.

\section{References}

Akbari ME, Haghighatkhah H, Shafiee M, et al (2012). Mammography and ultrasonography reports compared with tissue diagnosis - an evidence based study in Iran, 2010. Asian Pac J Cancer Prev, 13, 1907-10.

Alsanabani JA, Gilan W, Al Saadi A (2015). Incidence data for breast cancer among yemeni female patients with palpable breast lumps. Asian Pac J Cancer Prev, 16, 191-4.

Anderson BO, Yip CH, Smith RA, et al (2008). Guideline implementation for breast healthcare in low-income and middle-income countries: overview of the Breast Health Global Initiative Global Summit 2007. Cancer, 113, 222143.

Bang H, Zhao H (2012). Average cost-effectiveness ratio with censored data. Journal of Biopharmaceutical Statistics, 22, 401-15.

Carles M, Vilaprinyo E, Cots F, et al (2011). Cost-effectiveness of early detection of breast cancer in Catalonia (Spain). $B M C$ Cancer, 11, 2-11.

Davari M, Makarian F, Hosseini M, et al (2013). Evaluation of direct medical costs of breast cancer in Iran; Analysis of data from patients in a hospital specialized in cancer patients. Health Information Management Journal, 10, 1-10.

De Gelder R, Bulliard JL, WolfCD, et al (2009). Cost-effectiveness of opportunistic versus organized mammography screening in Switzerland. European Journal of cancer, 45, 127-38.

Eichler HG, Kong SX, Gerth WC, et al (2004). Use of costeffectiveness analysis in health-care resource allocation decision-making: how are cost-effectiveness thresholds expected to emerge?. Value In Health, 7, 518-28.

Farshbaf Khalili A, Shahnaz M, Qahvechi A, Jafar B (1999). Attitude of women referred to Tabriz health center regarding breast self-examination. Nursing and Midwifery Journal, Tabriz, 11, 38-46.

GLOBOCAN 2012 (IARC) Section of Cancer Information.

Greif M (2010). Mammographic screening for breast cancer: An invited review of the benefits and costs. The Breast, 19, 268-72.

Groot MT, Rob B, Groot CA, et al (2006). Costs and health effects of breast cancer interventions in epidemiologically different regions of Africa, North America, and Asia. The Breast Journal, 12, 81-90.

Hae KM, Park EC, Choi KS, et al (2013). The national cancer screening program for breast cancer in the Republic of Korea: is it cost-effective? Asian Pacific J Cancer Prev, 14, 2059-65.

Haghighat S (2013). Cost-effectiveness of mammography screening in iranian female. shahid beheshti university of medical sciences, Tehran.

Hoang Nguyen L, Laohasiriwong W, Frederick Stewart J, et al (2013). Cost-effectiveness analysis of a screening program for breast cancer in Vietnam. Value In Health Regional, 2, 21-8.

Jafari M, Nakhaee N, Goudarzi R, et al (2015). Participation of the women covered by family physicians in breast cancer screening program in Iran: 2014. Asian Pac J Cancer Prev, 16, 4555-61.

\section{Acknowledgements}


KI-BONG Y, Jeoung AK, Eun C, et al (2013). Is Mammography for breast cancer screening cost-effective in both western and asian countries? : results of a systematic review. Asian Pac J Cancer Prev, 14, 4141-49.

Lamberts OQ, Draisma G, Der Kinderen A, L Brown M, J De Koning H (2008). Breast cancer screening policies in developing countries: a cost-effectiveness analysis for India. JNCI, 100, 1290-300.

Laurens NM, Sten GZ, Gutiérrez-Delgado C, et al (2014). Costeffectiveness of breast cancer control strategies in central America: The cases of Costa Rica and Mexico. PLOS ONE, 9, 95836.

Mahboub-Ahari A, Pourreza A, Sari AA, et al (2014). Stated time preferences for health: a systematic review and meta analysis of private and social discount rates. J Res Health Sci, 14, 181-6.

Mandelblatt J, Freeman H, Winczewski D, et al (1997). The Costs and effects of cervical and breast cancer screening in a public hospital emergency room. Am J Public Hlth, 87, 1182-89.

Melnikow Joy, J.Tancredi D, Yang Z, et al (2013). Programspecific cost-effectiveness analysis: breast cancer screening policies for a safety-net program. Value Health, 16, 932-41.

Mousavi SM, Gouya MM, Ramazani R, et al (2009). Cancer incidence and mortality in Iran. Annals of Oncology, 20, 556-63.

Nahvijou A, Hadji M, BaratiMarnani A, et al (2014). A Systematic Review of Economic Aspects of Cervical Cancer Screening Strategies Worldwide: Discrepancy between Economic Analysis and Policymaking. Asian Pacific $J$ Cancer Prev, 15, 8229- 37.

Reza Z, Barooni M, Jafari M (2014). Assessing the costeffectiveness of breast cancer screening; a systematic review. Undergraduate. Health Care Management. 1-126.

Rojnik K, Naveršnik K, Mateovic'-Rojnik T, PrimicŽakelj M (2008). Probabilistic Cost-Effectiveness Modeling of Different Breast Cancer Screening Policies in Slovenia. Value in Health, 11, 139-48.

Sari AA, Ravaghi H, Mobinizadeh M, et al (2013). The Costutility analysis of PET-scan in diagnosis and treatment of non-small cell lung carcinoma in Iran. Iranian Journal of Radiology, 10, 1-7.

WHO (2008). Global Cancer Facts \& Figures. 2nd Edition, 1-37.

WHO (2003). Assessing the environmental burden of disease at national and local levels. Geneva, World Health Organization. WHO Environmental Burden of Disease Series, 1, 32-3.

Yip CH, Smith RA, Anderson BO (2008). Guideline implementation for breast healthcare in low- and middleincome countries: early detection resource allocation. Cancer, 113, 2244-56. 